\title{
Retinopathy of Prematurity in A Tertiary Care Hospital in Eastern Nepal
}

\author{
'Adhikari S, ' Badhu BP, ${ }^{2}$ Bhatta NK, ${ }^{2}$ Rajbhandari RS, ${ }^{2}$ Kalakheti BK \\ 'Department of Ophthalmology, B. P. Koirala Institute of Health Sciences, ${ }^{2}$ Department of Pediatrics and Adolescent Medicine, B. P. Koirala \\ Institute of Health sciences.
}

\section{ABSTRACT}

World Health Organization's Vision 2020 program has recognized Retinopathy of Prematurity (ROP) as an important cause of childhood blindness in industrialized and developing countries. In the last few years, it has been identified in many under developed countries as well, as a result of improved neonatal intensive care. In Nepal, ROP screening is carried out in a few tertiary hospitals but there is no published data on this disease. The purpose of this study was to find out the incidence, severity and risk factors of ROP among infants screened in a tertiary care hospital in the Eastern Region of Nepal.

A prospective cohort study was carried out in neonates with gestational age of 34 weeks or less and, or birth weight of $1700 \mathrm{gm}$ or less born over the period of one year. Dilated fundus examination of all babies was done by indirect ophthalmoscopy between 2-4 weeks after birth and followed up till the retinal vascularization was complete. Classification of ROP was done according to international classification (ICROP). Maternal and neonatal risk factors were also noted.

A total of 55 babies fulfilled the screening criteria. ROP was present in $25.45 \%(n=14)$ of the babies. Threshold disease was noted in $5.45 \%(n=3)$ of the babies screened. Low birth weight $(p<0.01)$ and low gestational age $(\mathrm{p}<0.01)$ was significantly associated with the incidence of ROP. Oxygen supplementation $(\mathrm{p}=<0.01)$ was an independent risk factor.

ROP screening should be performed in all preterm low birth weight infants where there is availability of good neonatal intensive care units. The examination should be intensified in those having risk factors like oxygen. Further studies in the other tertiary care hospitals in Nepal would help to establish the screening criteria for Nepalese infants.

\author{
Correspondence: \\ Dr Srijana Adhikari \\ Department of Ophthalmology \\ B. P. Koirala Institute of Health Sciences \\ Dharan, Nepal. \\ Email: srii_a@yahoo.com
}


Adhikari et al. Retinopathy of Prematurity in A Tertiary Care Hospital in Eastern Nepal

\section{INTRODUCTION}

Retinopathy of Prematurity (ROP) is defined as a bilateral vasoproliferative disease of the retina that occurs primarily in premature children but not exclusively so. ${ }^{1}$ World health Organization's vision 2020 program has identified ROP as an important cause of blindness in countries with low infant mortality rate. ${ }^{2}$ Studies suggest that ROP is more prevalent in premature neonates with very low birth weight. In industrialized countries, the survival rate of small preterm babies is high. Hence ROP is one of the major causes of childhood blindness in these countries. However, ROP is emerging as an important cause of blindness in children even in middle-income countries as those in Latin America, Asia and Eastern Europe. ${ }^{3}$ In developing countries, where preventable diseases of the cornea such as measles and avitaminosis are the major causes of blindness in childhood, retinal pathology accounts for a minority of all cases of childhood blindness. However, there are a few cases of ROP as a cause of blindness in these countries. ${ }^{4,5}$ This is mainly because of poor neonatal intensive care facilities in these countries.

Screening premature low birth weight infants for retinopathy of prematurity is carried out as a routine procedure in many countries of the world. However, this is still a very new concept in Nepal. Hospitals providing intensive care facilities are only a handful. The survival of low birth weight premature infants who meet the screening criteria is very rare. However, with better neonatal care facilities and experts, more and more premature infants are surviving in our hospitals as well. The aim of this study was to identify the incidence of ROP and possible risk factors and thereby to establish an appropriate screening program for ROP in our population.

\section{MATERIAL AND METHODS}

A hospital-based prospective cohort study was conducted at the Department of Ophthalmology and the Neonatal Unit of the Department of Pediatrics, in a tertiary hospital in the Eastern Region of Nepal. The study period was from April 2005 to July 2006. It was approved by the ethical and research committee of B. P. Koirala Institute of Health Sciences. Informed consent was taken from all the parents of babies who fulfilled the screening criteria.

The study population consisted of all preterm babies born during this period with the gestational age of 34 weeks or less and/or birth weight of $1700 \mathrm{gm}$ or less and who attended the follow up visits. The neonatal risk factors included in the study were multiple births, blood transfusion, septicemia, oxygen supplementation and hyperbilirubinaemia. Babies who died before they could be examined and those who did not complete the follow up examinations were excluded from the study.

\section{EXAMINATION SCHEDULE}

First examination: For babies born before 28 weeks, the first examination was done at four weeks post-natal age or at 32 weeks post-conceptional age, whichever was earlier. Babies born after 28 weeks of gestation were examined at two to three weeks after birth.

Follow up: If ROP was not detected at the initial examination, the infants were re-evaluated once every two weeks until their discharge from the hospital. Thereafter, they were examined every four weeks until vascularisation was complete. If ROP was detected, the examinations were performed weekly for stage 1-2 disease and more frequently for stage 3 disease, till the disease started resolving or progressed to the threshold stage. Babies showing evidence of regression were followed up weekly till vascularisation was complete. Babies progressing to the threshold stage were referred for treatment with cryotherapy or photocoagulation.

Pupils were dilated with a mixture of Phenylephrine $2.5 \%$ and Tropicamide $0.5 \%$ instilled 3 times at 10 minutes' interval about one hour before the scheduled examination. After decreasing the room illumination, indirect ophthalmoscopy was done by using a 20D Volk lens. A pediatric wire speculum was used to keep the eyelids apart. Indentation was done using a scleral depressor.

Classification of ROP was done according to the international classification of ROP (ICROP). Babies were grouped according to the stage and the zone of involvement of the disease. Babies with stage 1-2 disease were kept in ROP group I and those with more severe disease (stage 3-5) in ROP group II.

The data was analyzed by statistical package SPSS, version 11.5

\section{RESULTS}

The total number of babies born at 34 weeks of gestational age or less/and or weighing $1700 \mathrm{gm}$ or less at birth was 92 . However, 4 babies died before they could be examined. Three babies died after only 2 examinations, and 30 babies did not complete the follow-up examination. Thus only 55 babies fulfilled the inclusion criteria. There were 32 males and 23 females. Fourteen of the 55 babies (25.5\%) developed ROP. All babies had bilateral disease (Table 1 and 2).

Stage I disease was seen in 5 (35.71\%) babies, stage II in $6(42.85 \%)$ and stage III-V seen in $3(21.42 \%)$ babies.

In the classification according to the zone of involvement, $7(50 \%)$ babies were in zone III, 4 (28.57\%) in zone II and $3(21.42 \%)$ were in zone I group.

Birth weight in babies with ROP ranged from $700 \mathrm{gm}$ to $1500 \mathrm{gm}$ (mean1338.33 $\pm 194.61 \mathrm{gm}$ ). A lower birth weight was significantly associated with increased incidence of ROP $(p<0.01)$ (Table 3). 
Adhikari et al. Retinopathy of Prematurity in A Tertiary Care Hospital in Eastern Nepal

Table 1. Frequency and distribution of infants according to birth weight

\begin{tabular}{lc}
\hline BW in gm & Number (\%) \\
\hline $700-900$ & $1(1.8)$ \\
$>900-1100$ & $3(5.4)$ \\
$>1100-1300$ & $9(16.4)$ \\
$>1300-1500$ & $17(30.9)$ \\
$>1500-1700$ & $25(45.4)$ \\
Total & $55(100)$ \\
\hline
\end{tabular}

BW, birth weight

The gestational age of babies with ROP ranged from 26 to 32 wks (mean $30.50 \pm 1.53 w k s$ ). A lower gestational age significantly increased the incidence of $\operatorname{ROP}(p<0.01)$.

The cohort was followed up for the period ranging from 2-12 weeks. (Mean 5.6 weeks) (Table IV). Severe ROP requiring treatment (threshold to stage $\mathrm{V}$ disease) was seen exclusively in babies with birth weight $700 \mathrm{gm}$ to $1100 \mathrm{gm}$ and the gestational age of 26 to 30 weeks.

Among the neonatal risk factors, oxygen administration was significantly associated with incidence of ROP ( $p=<0.01)$. There were $16(29 \%)$ babies with twin-birth and $39(71 \%)$ were single. Hyperbilirubinemia requiring photo therapy was found in 42 (76\%). Twenty-five $(45 \%)$ babies required blood transfusion which included exchange transfusion. Neonatal septicemia was found in $65 \%$ of babies included in the study. On statistical analysis, except the oxygen administration, there was no significant association between the incidence of ROP and other neonatal risk factors.

Eleven babies with group I ROP showed regression without treatment while 3 babies with group II ROP required treatment with retinal photocoagulation.

\section{DISCUSSION}

Our study is the first reported study on retinopathy of prematurity in Nepalese infants. The incidence of ROP
Table 2. Frequency and distribution of infants according to the gestational age

\begin{tabular}{lcc}
\hline GA in Weeks & \multicolumn{2}{c}{ Number (\%) } \\
\hline $26-28$ & \multicolumn{2}{c}{$6(10.90)$} \\
$>28-30$ & \multicolumn{2}{c}{$10(18.18)$} \\
$>30-32$ & 22 & 40.00 \\
$>32-34$ & 17 & 30.90 \\
Total & 55 & 100 \\
\hline
\end{tabular}

GA, gestational age

in this study is similar to studies done in India, Taiwan and South Africa. However, the inclusion criteria differs in some of these studies. ${ }^{8,9,10,15,17}$ Studies suggest that every country should have their own criteria of screening according to the incidence and risk factors of ROP in that particular population and environment. We have included babies weighing $1700 \mathrm{gm}$ or less and/or gestational age 34 weeks or less.

In the CRYO ROP study, babies with birth weight of less than $1250 \mathrm{gm}$ and the gestational age of 28 weeks or less were included. ${ }^{6}$ A study from India had included babies with birth weight of $1700 \mathrm{gm}$ or less and the gestational age of 34 weeks or less. ${ }^{8}$

The association between ROP and supplemental oxygen has been confirmed by controlled trials and clinical studies. However, a safe level of oxygen administration has not been defined. In our study, oxygen administration was found to be an independent risk factor. This is similar to the study done in India. ${ }^{14}$ Sepsis, blood transfusion and multiple births have been found to be important risk factors for the development of ROP in many studies $8,14,15$ but not so in our study, in which there is no significant association between ROP and these factors.

Lower birth weight and gestational age were associated with the severity of the disease, similar to the studies in

Table 3. Incidence and severity of ROP in relation to birth weight

\begin{tabular}{|c|c|c|c|c|}
\hline \multirow[t]{2}{*}{ Birth Weight(gm) } & \multirow[t]{2}{*}{ Total Patients (\%) } & \multirow[t]{2}{*}{ ROP patients (\%) } & \multicolumn{2}{|c|}{ ROP group } \\
\hline & & & Group I & Group II \\
\hline $700-900$ & $1(1.81)$ & $1(100)$ & 0 & 1 \\
\hline$>900-1100$ & $3(5.40)$ & $3(100)$ & 1 & 2 \\
\hline$>1100-1300$ & $9(16.4)$ & $4(44.44)$ & 4 & 0 \\
\hline $1300-1500$ & $17(30.9)$ & $6(35.29)$ & 6 & 0 \\
\hline$>1500-1700$ & $25(45.45)$ & $0(0)$ & 0 & 0 \\
\hline Total & $55(100)$ & $14(25.45)$ & 11 & 3 \\
\hline
\end{tabular}


Adhikari et al. Retinopathy of Prematurity in A Tertiary Care Hospital in Eastern Nepal

Table 4. Incidence and severity of ROP in relation to gestational age

\begin{tabular}{lcccc}
\hline GA in weeks & Total patients (\%) & ROP patients (\%) & ROP group \\
\hline $26-28$ & $6(10.90)$ & $6(100)$ & 4 & 2 \\
$>28-30$ & $10(18.18)$ & $5(50)$ & 4 & 1 \\
$>30-32$ & $22(40.00)$ & $3(18.18)$ & 3 & 0 \\
$>32-34$ & $17(30.39)$ & $0(0)$ & 0 & 0 \\
Total & $55(100)$ & $14(25.45)$ & 11 & 3 \\
\hline
\end{tabular}

GA, gestational age

India and South Africa. ${ }^{15,17}$ In our study, only 3 babies reached threshold disease and required treatment.

\section{CONCLUSION}

This present study highlights the magnitude of the problem due to ROP in Nepalese preterm babies. The incidence is likely to increase as more preterm babies survive. The combined effort of neonatologists, obstetrician and the ophthalmologist is required in reducing childhood blindness due to ROP. We suggest that all preterm infants weighing $1500 \mathrm{gm}$ or less and the gestational age of 32 weeks or less should be screened at 4 weeks post-natal age.

\section{REFERENCES}

1. Nelson B, Harley H. Pediatric Ophthalmology. 4th ed. New York: Saunders; 2004

2. Gilbert C. Foster A. Childhood blindness in the context f vision 2020- the right to sight. Bull world health organization. 2001;79:227-32.

3. Gilbert C, Rahi J, Ection M, O Sullivan, Foster A. Retinopathy of prematurity in the middle income country. Lancet 1997;350:12-4.

4. O' Sullivan J, Gilbert C, Foster A. Causes of childhood blindness in South Africa. S Afr Med J 1997;87(12):16915 .

5. Fieldr AR, Shaw DF, Robinson J. Natural history of retinopathy of prematurity, a prospective study. Eye 1992;6:233-42.

6. Palmer EA, FlynnJT, Hardy RJ, et al. Incidence and early course of retinopathy of prematurity. Ophthalmology 1991;98:1628-40,

7. Darlow BA. Incidence of retinopathy of prematurity in New Zealand. Archive of disable in childhood 1998;63:1083-6.

8. Gopal I, Sharma T, Ramachandran S, Sanmugas sundaram R Asha V. Retinopathy of prematurity, a study. Indian journal of ophthalmology 1995; 43:5061.

9. Charan R Dogra, Gupta A, Narang A. The incidence of retinopathy of prematurity in a neonatal care unit. Indian journal of ophthalmology 1995;43:123-26.

10. Varughese S, Jain S, Gupta N, Singh S, Tyagi V, Puliyyel JM. Magnitude of problem of retinopathy of prematurity. Experience in a large maternity unit with a medium size, level 3 nursery. Indian journal of
Ophthalmology 2001;49:187-8.

11. International Committee for the Classification of Retinopathy of Prematurity. The International Classification of Retinopathy of Prematurity revisited. Arch Ophthalmol. 2005 Jul;123(7):991-9.

12. Gilbert C, Fielder A, Gordiillo L, et al. Characteristics of infants with severe retinopathy of preamaturity in countries with low, middle, and high level of development. Implication for the screening program. Pediatric 2005;115:518-25.

13. American Academy of Pediatrics, American Association for Pediatric Ophthalmology and Strabismus, American Academy of Ophthalmology. Screening examination of premature infants for retinopathy of prematurity. Pediatrics 2001;108:80911.

14. Gupta Ved P, Dhaliwal U, Sharma R, et al. Retinopathy of Prematurity-Risk factors. Indian Journal of Pediatrics 2004;71:887-92.

15. Yang K J Tsai CH, Lai CC, Chen TL. Retinopathy of prematurity: an evaluation in the Keelung area of Taiwan over a 10 year period. Chang Gunj Med Journal 2005, Jan;28(1):39-43.

16. William V good and early treatment for retinopathy of prematurity cooperative group. Final results of the early treatment of retinopathy of prematurity (ETROP) Randomized Trial. Trans American Ophthalmologic society 2004; 102:233-50.

17. I Mayet, C Cockinos. Retinopathy of prematurity in South African at a tertiary hospital: a prospective study. Eye 2006;20:29-31. 